\title{
Cirugía del sueño: Definición de una especialidad emergente, propuesta de una clasificación anatomofuncional integral e indicaciones
}

\section{Sleep surgery: Definition of an emerging specialty, proposal for a comprehensive anatomo-functional classification and indications}

\author{
Cristián E. Bachelet R. ${ }^{1}$, Felipe Castillo F. ${ }^{2}$, Sandra Zabala P. \\ René Pedraza A. ${ }^{4}$, Rodolfo Lugo S. ${ }^{5}$
}

'Programa de Medicina del Sueño, Departamento de Otorrinolaringología, Clínica Universidad de los Andes y Hospital San Martín de Quillota. Quillota, Chile. ¿Unidad de Sueño, Servicio de Otorrinolaringología, Hospital de Puerto Montt. Facultad de Medicina, Sede Patagonia, Universidad San Sebastián. Puerto Montt, Chile. ${ }^{3}$ Maple Respiratory Colombia, Clínica Marly JCG y Centro Médico Dalí. Bogotá, Colombia. ${ }^{4}$ Departamento de Otorrinolaringología y Cirugía Maxilofacial, Fundación Universitaria de Ciencias de la Salud - Hospital de San José de Bogotá. Bogotá, Colombia.

${ }^{5}$ Director de Programa en Desórdenes Respiratorios del Sueño, Ronquido y Rinología Aplicada-Universidad Autónoma de Nuevo León. Monterrey, México.

Los autores declaran no tener conflictos de interés.

Recibido el 14 de mayo de 2020. Aceptado el 7 de octubre de 2020 .

Correspondencia: Cristian E. Bachelet R. Departamento de Otorrinolaringología, Clínica Universidad de los Andes. Av. Plaza 2501, Las Condes. Santiago, Chile. Email: cbachelet@clinicauandes.cl

\section{Resumen}

Los trastornos respiratorios del sueño (ronquido primario y síndrome de apnea-hipopnea obstructiva del sueño) han sido tratados mediante múltiples modalidades a lo largo de la historia. Sin embargo, la cirugía de la vía aérea superior siempre ha estado presente, dando cabida a la aparición de múltiples técnicas para este fin. El estudio adecuado de los sitios anatómicos de estrechez o colapso de la vía aérea superior y sus contribuyentes (bajo el concepto de topodiagnóstico) y el mejor entendimiento de los mecanismos de acción de los diferentes procedimientos descritos, ha permitido el nacimiento de una nueva disciplina, dedicada al manejo quirúrgico planificado de este grupo de patologías: la cirugía del sueño.

Palabras clave: Trastornos respiratorios del sueño, topodiagnóstico, cirugía multinivel, cirugía del sueño.

\begin{abstract}
Sleep-related breathing disorders (primary snoring and obstructive sleep apnea-hypopnea syndrome) have been treated with multiple modalities throughout history. However, upper airway surgery has always been present, giving appearance of multiple techniques for this purpose. The adequate study of the anatomical sites of upper airway narrowness or collapse and its contributors (under the concept of topodiagnosis) and a better understanding of the different procedures, has allowed the birth of a new discipline, dedicated to a planned surgical management for this group of pathologies: sleep surgery.

Keywords: Sleep-related breathing disorders, topodiagnosis, multilevel surgery, sleep surgery.
\end{abstract}

Aspectos fisiopatológicos y concepto de cirugía del sueño

En los últimos años, se han dado importantes avances respecto al conocimiento de la fisiopatología de los trastornos respiratorios del sueño (TRS) y en particular del síndrome de apnea-hipopnea obstructiva del sueño (SAHOS), principalmente por estudios realizados por Eckert y cols. ${ }^{1,2}$ en los cuales se han descrito los siguientes endotipos fisiopatológicos:

- Vía aérea superior (VAS) estrecha o colapsable por una anatomía alterada (representado por una presión crítica de colapsabi- 
lidad de VAS -PCrit alta-): presente en el $100 \%$ de los pacientes con SAHOS, pero de forma variable en cuanto al grado de severidad y factores contribuyentes que determinan esta característica.

- Ineficacia de los músculos dilatadores de la VAS: presente en el 36\% de los pacientes con SAHOS.

- Control ventilatorio inestable (representado por un loop gain alto): presente en el $36 \%$ de los pacientes con SAHOS.

- Bajo umbral de despertar respiratorio: presente en el $37 \%$ de los pacientes con SAHOS.

Analizando de forma crítica las modalidades tradicionales de tratamiento del SAHOS, veremos que, en esencia, el objetivo principal es el mismo: el manejo de la estrechez o colapsabilidad de la VAS (reducción de la PCrit). Es así como podemos identificar las siguientes terapias como formas de reducir la PCrit: dispositivos de presión positiva de vía aérea (PAP), dispositivos orales de avance mandibular (DAM), cirugías de VAS, terapia posicional y la pérdida de peso ${ }^{1}$.

Dentro de los factores que determinan una VAS estrecha o colapsable por anatomía alterada, existen tres contribuyentes como los más estudiados:

- Sobrepeso y obesidad, ya que se generan depósitos grasos a nivel de las paredes laterales faríngeas y en el espesor de la lengua, estrechando la VAS.

- Alteraciones de los tejidos blandos de la VAS, ya que estos se consideran el contenido o las paredes de la VAS.

- Alteraciones dentomaxilofaciales, ya que el esqueleto maxilofacial se considera el continente de la VAS.

Si bien se han desarrollado algunas estrategias para intentar manejar los otros endotipos fisiopatológicos del SAHOS, todas se consideran más recientes o con relativa menor evidencia, por ejemplo: terapia miofuncional orofaríngea $^{3}$ e implantes de nervio hipogloso ${ }^{4}$ (para el manejo de la ineficacia muscular de VAS), oxigenoterapia y acetazolamida ${ }^{5}$ (para el manejo del control ventilatorio inestable) y ciertos hipnóticos ${ }^{6}$ (para el manejo del bajo umbral de despertar respiratorio).
Teniendo en cuenta estos aspectos tan importantes, es que podemos entender el concepto de cirugía del sueño como una disciplina que integra el uso racional de un grupo de procedimientos invasivos de diversa índole (procedentes de la otorrinolaringología, cirugía maxilofacial y cirugía bariátrica), con el objetivo de tratar pacientes con TRS (principalmente SAHOS y ronquido primario), al intentar reducir la estrechez o colapsabilidad de la VAS.

$\mathrm{Al}$ igual que otras estrategias de tratamiento en este tipo de patología, la cirugía del sueño no pretende ser una cura definitiva en todos los pacientes, dada la naturaleza crónica y multifactorial de los TRS. Más bien, es una modalidad de terapia que busca el crear una VAS lo más adecuada posible (en tamaño y estabilidad) para cada paciente en particular. De ahí que la cirugía del sueño se considera como parte del arsenal de manejo multidisciplinario de los TRS. En ese contexto, según el objetivo deseado, puede plantearse la cirugía de sueño como terapia principal, pero también como adyuvante para facilitar el uso de dispositivos que reduzcan la PCrit (PAP o DAM), concepto conocido como cirugía de adaptación de dispositivos (PAP/DAM).

Se sabe que en los pacientes con TRS, la VAS puede tener colapsos en uno o múltiples sitios o niveles anatómicos, sin embargo, los últimos estudios de análisis de VAS han mostrado colapso multinivel en más del 70\% de los casos $^{7}$. Inicialmente, Fujita clasificó los niveles de colapso en retropalatal y retrolingual ${ }^{8}$, lo que llevó a clasificar las cirugías del sueño en palatinas e hipofaríngeas. No obstante, el término de cirugía hipofaríngea resulta ser anatómicamente impreciso, al incluir procedimientos que involucraban la lengua o la laringe.

Con el reconocimiento del efecto anatómico de diversas técnicas quirúrgicas en correlación con nuevas definiciones en el análisis de VAS, resulta más preciso dividir posibles colapsos de la VAS en los siguientes niveles (Tabla 1):

- Nasal-nasofaríngeo: La nariz (con todos sus componentes) y la nasofaringe se comportan en la práctica como una unidad, ya que es todo el segmento semirrígido inicial de la VAS, en donde se genera gran parte de su resistencia. 


\begin{tabular}{|ll|}
\hline \multicolumn{2}{|l|}{ Tabla 1. Niveles de colapso de las VAS en TRS } \\
\hline Nivel & Subsitios anatómicos \\
\hline Nasal-nasofaríngeo & $\begin{array}{l}\text { Válvula nasal externa e interna } \\
\text { Apertura piriforme y piso nasal } \\
\text { Septum } \\
\text { Cornetes } \\
\end{array}$ \\
& $\begin{array}{l}\text { Meatos y senos paranasales } \\
\text { Nasofaringe }\end{array}$ \\
Orofaríngeo retropalatal & $\begin{array}{l}\text { Velo del paladar } \\
\text { Paredes laterales orofaríngeas }\end{array}$ \\
Orofaríngeo retrolingual- & $\begin{array}{l}\text { Base de lengua } \\
\text { hipofaríngeo }\end{array}$ \\
Laríngeo & Earedes laterales hipofaríngeas \\
& Aritenoides \\
\hline
\end{tabular}

- Orofaríngeo retropalatal: Este sector integra la velofaringe y las paredes laterales orofaríngeas, que en la práctica dependen de la musculatura del paladar blando, comportándose como una unidad.

- Orofaríngeo retrolingual-hipofaríngeo: Este sector integra la base de la lengua (parte de la orofaringe) y las paredes laterales hipofaríngeas, que suelen colapsar como una sola unidad.

- Laríngeo: Si bien este sector puede colapsar en dependencia de una estrechez a nivel orofaríngeo retrolingual-hipofaríngeo, se han demostrado colapsos completamente independientes en este sitio.

Dichos niveles anatómicos de colapso no sólo se afectan por problemas de los tejidos blandos de la VAS en sí mismos, sino que podemos verlos afectados también por los otros factores contribuyentes a alteraciones anatómicas:

- Alteraciones dentomaxilofaciales:

- Del maxilar superior: este hueso, al rodear las fosas nasales y tener inserciones del paladar blando, teóricamente puede generar a su vez estrechez en los niveles nasal-nasofaríngeo y orofaríngeo retropalatal ${ }^{9}$.

- De la mandíbula: este hueso, al tener inserciones de la musculatura suprahioídea y de los músculos constrictores faríngeos superior y medio, teóricamente puede generar estrechez en niveles orofaríngeo retropalatal y orofaríngeo retrolingual-hipofaríngeo $^{10}$.
- Sobrepeso y obesidad:

- Al haberse demostrado depósitos grasos en las paredes laterales faríngeas y en el espesor de la lengua, teóricamente generan estrechez en niveles orofaríngeo retropalatal y orofaríngeo retrolingual-hipofaríngeo ${ }^{11}$.

Hoy en día se utiliza el término de topodiagnóstico del colapso de la VAS, a la determinación de los principales sitios o niveles de colapso en la VAS, así como sus contribuyentes a la alteración anatómica. Esto se logra después de un adecuado examen físico de la VAS, así como también de una serie de estudios complementarios. Esto ha podido crear un cambio de paradigma en la forma de hacer cirugía del sueño, determinando una mejor selección del paciente y de las técnicas a emplear en cada caso, según las características individuales de la VAS.

La cirugía del sueño estuvo desprestigiada durante mucho tiempo, puesto que en sus inicios los resultados eran relativamente pobres $^{12,13}$. Esto probablemente se debía a la práctica de estos procedimientos por ensayo y error, sin un análisis exhaustivo de la VAS antes de definir la conducta. Por otro lado, las cirugías inicialmente tenían una intención más ablativa que reconstructiva, lo que determinaba una mayor frecuencia de complicaciones funcionales a largo plazo ${ }^{14}$.

Afortunadamente, con la evolución de métodos de análisis de VAS y técnicas quirúrgicas, se ha dado un escenario donde la cirugía del sueño está en situación emergente y muy dinámica en cuanto a los protocolos de manejo. De este modo, podemos entender por qué se ha logrado posicionar esta especialidad quirúrgica en los últimos años con un rol importante dentro de las alternativas de tratamiento de los TRS, sobre todo en aquellos pacientes que rechazan o no toleran adecuadamente el uso habitual de dispositivos para reducir el colapso de la VAS (como PAP o DAM).

\section{Clasificación de la cirugía del sueño}

Uno de los factores que hace complejo el aprendizaje de la cirugía del sueño, es que existen múltiples técnicas quirúrgicas mencionadas en la literatura para tratar los TRS. Es por esto, que se propone una nueva clasificación 
de estas intervenciones, de modo de agruparlas según su mecanismo de acción, sitios anatómicos a los que están dirigidos y gesto principal de la técnica quirúrgica, facilitando así su comprensión (Tabla 2).

- Cirugía de bypass de VAS: Su intención es crear una derivación de la VAS, desviándola de los colapsos superiores sin tener que intervenir sobre ellos directamente. Su único exponente es la traqueostomía ${ }^{8,15}$.

- Cirugía de modificación anatómica de VAS: $\mathrm{Su}$ intención es intervenir directamente sobre los contribuyentes que hacen que la
VAS sea estrecha o colapsable del punto de vista anatómico, por lo tanto, apunta directamente a reducir la PCrit ${ }^{1,16}$.

- Cirugía de estimulación de tono muscular de VAS: Su intención es reducir la colapsabilidad de la VAS a través del aumento de la eficacia de sus músculos dilatadores. $\mathrm{Su}$ único exponente es el estimulador de nervio hipogloso ${ }^{17}$.

En términos generales, parece adecuado seleccionar las técnicas quirúrgicas a utilizar según:

\section{Tabla 2. Clasificación integral de procedimientos en cirugía del sueño}

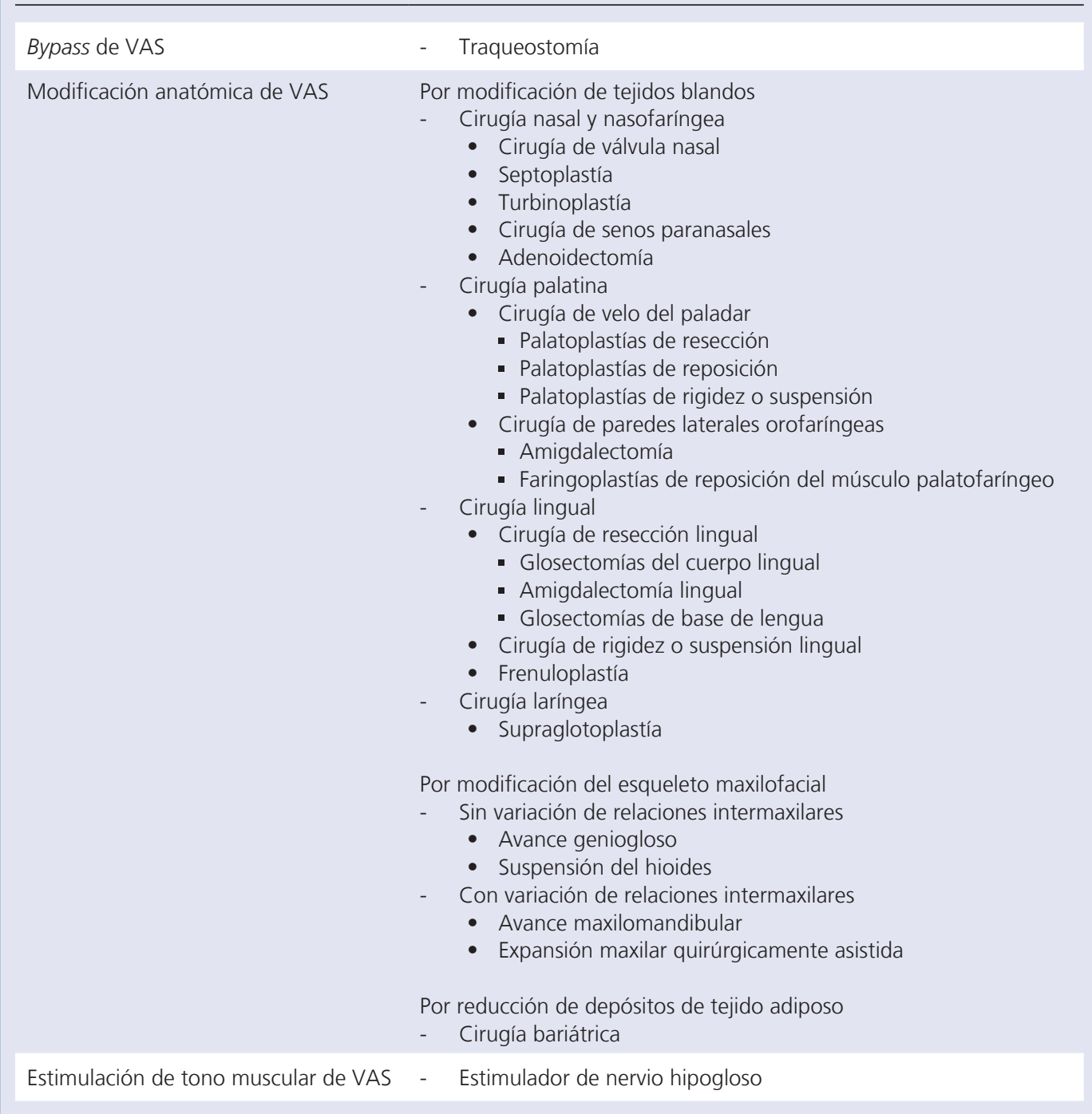


- Condiciones generales del paciente (edad, comorbilidades y preferencias).

- Topodiagnóstico de VAS (niveles afectados y factores contribuyentes).

- Magnitud del cuadro (índice de apnea-hipopnea-IAH-, oxigenación, etc.).

- Objetivo de la intervención (como terapia principal o para adaptar dispositivos).

- Experiencia del cirujano.

\section{A) Cirugía de bypass de VAS}

\section{Traqueostomía}

La traqueostomía fue la primera opción quirúrgica descrita en casos de SAHOS severo y la única que asegura una resolución del cuadro prácticamente completa ${ }^{15}$. En general está indicada de forma excepcional y como último recurso en SAHOS severo asociado a otras enfermedades de base que puedan agravarse de forma importante, pudiendo comprometer la vida del paciente, siempre y cuando otras terapias no hayan sido toleradas o efectivas ${ }^{18}$.

Como en estos casos se suele plantear por períodos prolongados o incluso de forma permanente, se ha empleado una modificación de la técnica con colgajos de piel que se introducen por el estoma (skin-lined tracheostomy) $)^{19}$ para producir una cobertura que permita una mejor maduración y estabilidad del estoma en el tiempo, con menos granulación y mejor apertura.

\section{Tabla 3. Criterios generales de selección para cirugía multinivel de VAS}

Paciente adulto

- Idealmente edad <60-65 años

SAHOS o ronquido primario

- Idealmente IAH < 60-65 (salvo si hay alteración severa de tejidos blandos de VAS)

Alteración de tejidos blandos de VAS relevante

- Paciente ideal pero no siempre presente

Ausencia de exceso importante de tejido adiposo Idealmente índice de masa corporal (IMC) $<30-35 \mathrm{~kg} / \mathrm{m}^{2} \mathrm{y}$ circunferencia de cuello $<43-45 \mathrm{~cm}$

Ausencia de anomalía dentomaxilofacial severa

- Valorar según criterios generales de cirugía ortognática

Fracaso de medidas no quirúrgicas

Por rechazo, intolerancia o mala respuesta

\section{B) Cirugía de modificación anatómica de VAS}

\section{b.1) Cirugía por modificación de tejidos blandos de VAS}

Salvo excepciones, las cirugías de este grupo no suelen realizarse de forma independiente en adultos. La metódica actual es que, dependiendo del topodiagnóstico de la VAS, lo más frecuente es asociarlas entre sí cuando la intención es plantear estos procedimientos como terapia principal para TRS. En este contexto se le suele llamar cirugía multinivel de VAS. Algunos criterios generales de selección están listados a continuación (Tabla 3 ).

Según los niveles que se van a intervenir, existen múltiples opciones quirúrgicas a saber:

\section{b.1.1) Cirugía nasal y nasofaríngea}

Este ámbito involucra una serie de procedimientos para mejorar la obstrucción a nivel nasal-nasofaríngeo como factor predisponente al colapso de VAS. Como cirugía exclusiva, si bien es capaz de reducir la intensidad del ronquido, no suele modificar mayormente el $\mathrm{IAH}^{20}$. Sin embargo, la importancia de la nariz está dada por ser el sitio donde se produce gran parte de la resistencia de la VAS, de modo que, si esta resistencia aumenta, por modelo de Starling ${ }^{21}$ se puede explicar una mayor probabilidad de colapsos en los sitios restantes de la VAS.

También puede ser planteada como cirugía exclusiva en contexto de cirugía de adaptación de dispositivos (PAP/DAM), ya que la obstrucción nasal puede impedir el uso adecuado de una interfase o mascarilla nasal en caso de CPAP (debiendo adaptar interfase oronasal, que suele dar mayor IAH residual y peor tolerancia a largo plazo) o también puede dificultar el uso adecuado de un DAM (que obliga a mantener la boca semicerrada durante su uso, incomodando a pacientes respiradores orales) ${ }^{22}$.

En general, los pacientes que más se benefician son aquellos donde se logra transformar de respirador oral a respirador nasal. Se pueden clasificar en:

\section{- Cirugía de válvula nasal}

Para el manejo de colapsos a nivel de la válvula nasal externa e interna. La principal cirugía representante es la rinoplastía fun- 
cional (con diversas técnicas dependiendo si el colapso se produce de forma dinámica o estática), por lo que también puede tener implicancias estéticas. También se han descrito técnicas con suturas de suspensión y rigidez por radiofrecuencia ${ }^{23}$ (principalmente para colapsos dinámicos de válvula nasal interna). Existe controversia en cuanto a que este grupo de procedimientos sí logre un mayor impacto en reducción de IAH, comparado con otras cirugías nasales.

\section{- Septoplastía}

Para el manejo de las desviaciones cartilaginosas y óseas del septum nasal, o como parte de un procedimiento de rinoplastía funcional cuando el septum colapsa las válvulas nasales o para obtención de injertos.

\section{- Turbinoplastía}

Principalmente para el manejo de la hipertrofia de cornetes inferiores refractaria a tratamiento médico. Esta puede ser a expensas de su porción mucosa periférica o de su porción ósea central.

\section{- Cirugía de senos paranasales}

Principalmente la cirugía endoscópica nasal para el manejo de patología rinosinusal crónica o recurrente refractaria a tratamiento médico, que pudiera ser contribuyente importante en la obstrucción nasal. Un ejemplo importante para TRS es la rinosinusitis crónica con pólipos.

\section{- Adenoidectomía}

Principal cirugía realizada a nivel de rinofaringe, en caso de hipertrofia adenoidea. Si bien es más frecuente de realizar en niños, ocasionalmente puede hallarse algún grado de hipertrofia persistente en adultos respiradores orales crónicos.

\section{b.1.2) Cirugía palatina}

Este grupo de procedimientos están dirigidos al manejo de los colapsos de VAS a nivel orofaríngeo retropalatal. En términos generales, la evolución de estas técnicas ha ido dejando de lado las técnicas con intención ablativa, por nuevos procedimientos de reposición (principalmente de reposición del músculo palatofaríngeo $)^{24}$ debido a la alta tasa de complicaciones posoperatorias relevantes a largo plazo en las primeras (disfagia persistente, insuficiencia velofaríngea y estenosis velofaríngea) $)^{25}$ :

\section{- Cirugía del velo del paladar}

Se encarga principalmente del manejo de la velofaringe, sobre todo a nivel de la línea media palatina. No obstante, existen múltiples variaciones de dichas técnicas para aumentar el manejo de sectores laterales con extensión a paredes laterales faríngeas. Se pueden clasificar a su vez en:

\section{- Palatoplastías de resección}

- En general están en desuso. En este grupo se busca el retiro de porciones musculares y mucosas del velo del paladar. Se incluyen: uvulectomía (hoy en día se prefiere realizar de forma parcial en su porción no muscular, en caso de que esté elongada), resección parcial palatina de Quesada-Perelló ${ }^{26}$, uvulopalatofaringoplastía de Ikematsu-Fujita (UPPP) ${ }^{27}$ con sus modificaciones iniciales, resección intrapalatina de Crestinu ${ }^{28}$ y uvulopalatoplastía asistida con láser de Kamami $(\text { LAUP })^{29}$.

- Palatoplastías de reposición

- En este grupo se movilizan porciones importantes de músculo y mucosa mediante colgajos, pudiendo remover parte de mucosa o hueso palatino, pero sin resección muscular. Se incluyen: colgajo uvulopalatal de Powell-Riley ${ }^{30}$, avance transpalatino de Woodson ${ }^{31}$ y zeta-palatoplastía de Friedman (ZPP) ${ }^{32}$.

- Palatoplastías de rigidez o suspensión

- En este grupo se pretende generar fibrosis o sólo tracción de los tejidos, en ocasiones dependiendo de resección mucosa limitada, pero sin resección muscular. Se incluyen: radiofrecuencia intersticial del paladar, escleroterapia, CAPSO (cautery-assisted palatal stiffening operation $)^{33}$, palatoplastía anterior de Pang ${ }^{34}$, cortinas romanas de Mantovani $i^{35}$ (con suturas barbadas), injertos palatinos e implantes palatinos (en desuso por extrusiones). 
- Cirugía de paredes laterales orofaringeas Se pueden clasificar a su vez en:

- Amigdalectomía.

- Se puede realizar de forma exclusiva (en caso de amígdalas palatinas hipertróficas) o como paso previo a una cirugía de reposición del músculo palatofaríngeo.

- Faringoplastías de reposición del músculo palatofaríngeo.

- Dentro de las cirugías palatinas, son las más popularizadas actualmente. Son capaces de manejar las paredes laterales orofaríngeas y, además, logran avanzar la línea media palatina o la velofaringe. Se basan en realizar una disección meticulosa para crear un colgajo del músculo palatofaríngeo (pilar amigdalino posterior), para su reposición en una ubicación más lateral, superior y anterior. En este grupo se incluyen: faringoplastía lateral de Cahali $^{24}$, faringoplastía de expansión de esfínter de Pang-Woodson ${ }^{36}$, faringoplastía de recolocación de $\mathrm{Li}^{37}$ y faringoplastía de reposición barbada de Vicini ${ }^{38}$. Se han descrito también múltiples modificaciones de estas técnicas para mejorar su manejo de la línea media palatina ${ }^{39-41}$.

\section{b.1.3) Cirugía lingual}

Este grupo de procedimientos están dirigidos a mejorar colapsos de VAS a nivel orofaríngeo retrolingual-hipofaríngeo, principalmente por colapsos de la base de lengua. Se pueden clasificar a su vez en los siguientes tipos:

- Cirugía de resección lingual

Se pueden clasificar a su vez en:

- Glosectomías del cuerpo lingual

- En este grupo se busca el retiro de porciones musculares y/o mucosas de la lengua, desde un abordaje por delante de la $\mathrm{V}$ lingual (aunque pueden extenderse a base de lengua). Se incluyen: glosectomía anterior, glosectomía media submucosa de Woodson ${ }^{42}$, linguoplastía submucosa de Robinson ${ }^{43}$, SMILE (submucosal minimally invasive lingual excision $)^{44} \mathrm{y}$ CobLAMO (coblation-assisted Lewis and MacKay operation $)^{45}$.
- Amigdalectomía lingual.

- La remoción parcial o total de las amígdalas linguales se puede plantear de forma exclusiva (en caso de amígdalas linguales hipertróficas) o como paso previo a una glosectomía de base de lengua. Se han descrito diversos métodos, pero los más popularizados actualmente son: radiofrecuencia ${ }^{46}$ y cirugía transoral robótica $(\mathrm{TORS})^{47}$.

- Glosectomías de base de lengua.

- En este grupo se busca el retiro de porciones musculares y/o mucosas de la lengua, principalmente por detrás de la $\mathrm{V}$ lingual. Se incluyen: glosectomía media posterior de Fujita ${ }^{48}$, linguoplastía de Woodson ${ }^{49}$, glosectomía submucosa percutánea de Robinson $^{50}$, reducción transcervical de Chabolle ${ }^{51}$, CELL (coblation endoscopic lingual lightening) ${ }^{52}$, TORS ${ }^{47-53}$ y Robo-Cob de Bahgat ${ }^{54}$.

\section{- Cirugía de rigidez o suspensión lingual}

En este grupo se busca generar fibrosis o sólo tracción de los tejidos linguales, sin resección muscular o mucosa. Se incluyen: radiofrecuencia intersticial de lengua ${ }^{55} \mathrm{y}$ suspensión lingual (mediante suturas que pueden acompañarse de mecanismos de anclaje a la sínfisis mandibular $)^{56}$.

\section{- Frenuloplastía}

La sección del frenillo lingual está indicada en caso de anquiloglosia (frenillo lingual corto). Si bien, este factor se ha considerado un factor relevante sobre todo en niños por posibles alteraciones en crecimiento maxilofacial, también se ha utilizado en adultos, en conjunto con terapia miofuncional ${ }^{57}$.

\section{b.1.4) Cirugía laríngea}

Este grupo de procedimientos está dirigido al manejo de los colapsos de VAS a nivel laríngeo. Está representada por las supraglotoplastías, principalmente, la epiglotectomía parcial (aunque se ha descrito también la epiglotopexia y la rigidez epiglótica). Se realiza en casos de epiglotis flácida o laringomalacia relacionada a sueño, demostrable principalmente en endoscopía de sueño inducido por fármacos (DISE). Existen múltiples modalida- 
des de ejecución: técnica fría, electrocauterio, radiofrecuencia, TORS, etc.

Además, se cree que la epiglotectomía en pacientes con epiglotis flácida que colapse en sentido anteroposterior (trapdoor epiglottis), puede funcionar como cirugía de adaptación de $P A P$, ya que un CPAP podría inducir colapso epiglótico en estos casos por la misma presión de aire, generando mala tolerancia al equipo ${ }^{58}$. De forma excepcional en adultos, se ha descrito la necesidad de resección parcial de los cartílagos aritenoides por ser contribuyentes del colapso (descrita mayormente en niños con laringomalacia relacionada a sueño) ${ }^{59}$.

\section{b.2) Cirugía por modificación del esqueleto maxilofacial}

Este grupo de cirugías basa su impacto en la VAS por la tracción y estabilización de diversos grupos musculares a nivel faríngeo, a través de la reposición de segmentos óseos con el consecuente arrastre de sus inserciones musculares $^{60,61}$. Se puede a su vez clasificar según si modifican o no las relaciones intermaxilares, es decir, la posición que tienen el maxilar superior y la mandíbula entre sí y respecto al resto del esqueleto craneofacial.

\section{b.2.1) Cirugía esquelética sin variación de relaciones intermaxilares}

Este grupo de procedimientos están dirigidos al manejo de los colapsos de VAS en los niveles orofaríngeo retrolingual-hipofaríngeo y laríngeo, pudiendo teóricamente estabilizar la lengua, paredes laterales hipofaríngeas y epiglotis ${ }^{62,63}$. Estas cirugías rara vez se realizan de forma aislada, sino que suelen ser parte de una cirugía multinivel de VAS o como complemento a un avance maxilomandibular. Se pueden distinguir a su vez los siguientes tipos:

\section{- Avance geniogloso}

Este procedimiento pretende avanzar la inserción principal del músculo geniogloso (principal músculo dilatador de la VAS), que va desde la apófisis geni (ubicados en la sínfisis mandibular por su cara lingual) al dorso de la lengua y hueso hioides. Se puede clasificar a su vez en:

- Avance geniogloso propiamente tal

- Este procedimiento busca hacer un seg- mento óseo (generalmente rectangular) en la sínfisis mandibular a la altura de la apófisis geni, de modo de traccionar y fijar dicha inserción en una posición más anterior. Se han descrito múltiples diseños del segmento y formas de fijación.

- Genioplastía

- Esta cirugía pretende avanzar el mentón en bloque, lo cual, a diferencia del procedimiento anterior, también tiene implicancias estéticas. Existe un beneficio adicional teórico en la VAS por traccionar no sólo el músculo geniogloso, sino también otros grupos musculares (geniohioídeo, digástrico y miohioídeo). También se han descrito múltiples diseños de osteotomías y formas de fijación.

\section{- Suspensión del hioides}

Este procedimiento busca traccionar el hueso hioides, para estabilizar grupos musculares suprahioídeos (geniogloso, geniohioídeo, hiogloso y milohioídeo) así como el músculo constrictor medio faríngeo. Se puede clasificar a su vez en:

\section{- Opción hio-tiroidea}

- Este procedimiento busca traccionar el hioides en vector inferior, haciendo una pexia entre el hioides y el borde superior del cartílago tiroides de la laringe. Se considera más fácil de ejecutar y estéticamente mejor que la opción hio-mandibular ${ }^{64}$.

- Opción hio-mandibular

- Este procedimiento busca traccionar el hioides en vector superior, acercándolo al reborde inferior de la mandíbula mediante suturas con anclajes óseos (similares a los usados en la suspensión lingual). Si bien es técnicamente más complejo, este vector teóricamente expande y estabiliza mejor la $\mathrm{VAS}^{65}$.

\section{b.2.2) Cirugía esquelética con variación de relaciones intermaxilares}

Este grupo de procedimientos están dirigidos al manejo de los colapsos de VAS de forma multinivel en sí mismos (teóricamente pueden estabilizar múltiples sitios). Estas cirugías se consideran formas específicas de planificación de cirugía ortognática, por lo que también 
tienen implicancias en la oclusión dental y estética facial. Se pueden distinguir a su vez los siguientes tipos:

\section{- Avance maxilomandibular}

Busca estabilizar la VAS mediante la tracción de múltiples estructuras musculares, con impacto teórico en los niveles orofaríngeo retropalatal, orofaríngeo retrolingual-hipofaríngeo y laríngeo. Su principal efecto demostrado es a nivel de las paredes laterales faríngeas ${ }^{10}$ (a nivel orofaríngeo e hipofaríngeo). Se busca realizar osteotomías que permitan movimientos de avance y/o rotación antihoraria tanto del maxilar superior como de la mandíbula, idealmente en pacientes con SAHOS y anomalías dentomaxilares que pudieran beneficiarse de dichas maniobras (retrognatismo mandibular, retrognatismo maxilar superior y exceso vertical maxilar superior con crecimiento hiperdivergente). Los principales criterios de selección están listados a continuación (Tabla 4).

- Expansión maxilar quirúrgicamente asistida.

Este procedimiento tiene efecto demostrado en la VAS principalmente a nivel nasal-nasofaríngeo, ya que busca expandir la apertura piriforme y el piso nasal, dependientes de los huesos maxilar superior y palatino (que forman el paladar duro). Tiene un efecto teórico también sobre el velo del paladar (inserto el borde posterior del paladar duro) y sobre la

Tabla 4. Criterios generales de selección para avance maxilomandibular

Paciente adulto

- Ideal edad < 60-65 años

SAHOS o ronquido primario

Habitualmente $\mathrm{IAH} \geq 15-30$ (salvo si hay alteración severa dentomaxilofacial)

Anomalía dentomaxilofacial relevante

- Paciente ideal pero no siempre presente

Ausencia de exceso importante de tejido adiposo Ideal IMC $<35-40 \mathrm{~kg} / \mathrm{m}^{2}$

Ausencia de alteración de tejidos blandos de VAS severa Principalmente hipertrofia de amígdalas palatinas o linguales

Fracaso de medidas no quirúrgicas Por rechazo, intolerancia o mala respuesta lengua (al permitir mejor entrada de ella en la cavidad oral después de ampliar el paladar duro). Habitualmente se logra mediante osteotomías en el maxilar superior y el uso de dispositivos expansores, diseñados para aumentar su diámetro transverso, bajo principios de distracción osteogénica. Si bien también es factible de realizar expansión mediante segmentación del maxilar cuando la discrepancia oclusal no es muy grande, tiene desventajas teóricas respecto al método por distracción osteogénica en cuanto al impacto que podría generar a nivel del piso nasal. Está indicado idealmente en pacientes con SAHOS y anomalías dentomaxilares que pudieran beneficiarse de esta técnica (déficit transverso maxilar superior). Se han descrito varias modalidades: SARME/SARPE (surgically assisted rapid maxillary/palatal expansion) ${ }^{66}$, DOME (distraction osteogenesis maxillary expansion) ${ }^{67}$ y EASE (endoscopically assisted surgical expansion $)^{68}$. Los principios de esta técnica tradicionalmente han sido usados sin apoyo quirúrgico en niños (rapid maxillary expansion-RME- $)^{69}$ y recientemente también en adultos jóvenes (microimplant assisted rapid palatal expansion-MARPE- $)^{70}$.

\section{b.3) Cirugía por reducción de depósitos de tejido adiposo}

\section{Cirugía bariátrica}

Este grupo de procedimientos busca apoyar tratamientos para baja de peso, de modo de reducir los depósitos de grasa que pueden estrechar la VAS, demostrados en paredes laterales faríngeas y en el espesor de la lengua. Por lo tanto, tiene efecto teórico principalmente en los niveles orofaríngeo retropalatal y orofaríngeo retrolingual/hipofaríngeo. Está indicada en pacientes adultos con SAHOS e $\mathrm{IMC} \geq 35 \mathrm{~kg} / \mathrm{m}^{2.71}$.

\section{C) Cirugía de estimulación de tono muscular de VAS}

\section{Implante de nervio hipogloso}

Este grupo de dispositivos implantados quirúrgicamente, buscan estimular directamente las ramas del nervio hipogloso (nervio motor encargado de inervar la musculatura intrínseca de la lengua). Esto ha demostrado una activación de la actividad muscular lingual principal- 
mente, pero también, en menor cuantía, de la musculatura del paladar. Es por esto, que está orientado principalmente para colapsos del nivel orofaríngeo retrolingual-hipofaríngeo, sobre todo a expensas de la base de lengua ${ }^{17,72}$. Existen reportes de cuatro dispositivos que difieren en zonas de implantación y modalidad de acción, no obstante, el que tiene mayor evidencia en la literatura es el implante Inspire. Este dispositivo depende de tres incisiones para la inserción de sus partes: una cervical para la guía de estimulación (en contacto con el nervio hipogloso), una subclavicular para el marcapaso generador de pulso y una intercostal para la guía de sensor respiratorio. Los criterios de selección usados para el implante Inspire, se mencionan a continuación (Tabla 5).

\section{Conclusión}

Con todos los argumentos descritos, la cirugía del sueño se considera una especialidad emergente, en particular dentro del ámbito de la otorrinolaringología. Ésta integra conceptos de otras especialidades para la selección de la mejor terapia en cada paciente en particular, complementando una visión amplia de diversas alternativas quirúrgicas y no quirúrgicas. La comprensión de los diversos endotipos fisiopatológicos y las nuevas estrategias para el topodiagnóstico del colapso de la VAS en pacientes portadores de TRS, han aumentado la precisión en las indicaciones tanto para la selección del paciente quirúrgico, así como también del tipo de cirugía a realizar.

En esta revisión, se ha expuesto un resumen de las bases fisiopatológicas que avalan a la cirugía del sueño de manera coherente dentro del manejo multidisciplinario de los TRS. También se ha realizado una propuesta de clasificación integral que engloba las diversas técnicas quirúrgicas disponibles a la fecha en un sentido anatómico y funcional. Los TRS son altamente frecuentes y desde la otorrinolaringología debemos aportar nuestra visión con un mayor entendimiento y competencias en su diagnóstico y tratamiento. Al ser un área en pleno desarrollo, es tarea de las nuevas generaciones de especialistas el fomentar su formación e investigación en este ámbito, para generar un aporte significativo dentro de
Tabla 5. Criterios generales de selección para implante Inspire

De inclusión

- SAHOS con $I A H \geq 15 y<65$

- Rechazo, intolerancia o mala respuesta a CPAP

De exclusión

- $\quad$ IMC $\geq 32-35 \mathrm{~kg} / \mathrm{m}^{2}$

- Índice de apneas centrales (IAC) y mixtas (IAM) $\geq 25 \%$ del IAH total

DISE que muestre colapso concéntrico completo velofaríngeo

Necesidad futura de estudios de resonancia magnética

- Comorbilidad psiquiátrica o de sueño (ej: insomnio severo) relevante

- Patología congénita o motora relevante de la lengua

- Embarazo

- Enfermedad sistémica severa o baja expectativa de vida ( $<12$ meses)

los equipos multidisciplinarios dedicados al manejo integral de estos pacientes.

\section{Bibliografía}

1. Eckert DJ, White DP, Jordan AS, Malhotra A, Wellman A. Defining phenotypic causes of obstructive sleep apnea. Identification of novel therapeutic targets. Am J Respir Crit Care Med. 2013;188(8):996-1004. doi: 10.1164/rccm.2013030448OC.

2. Eckert DJ. Phenotypic approaches to obstructive sleep apnoea - New pathways for targeted therapy. Sleep Med Rev. 2018;37:45-59. doi: 10.1016/j. smrv.2016.12.003

3. Camacho M, Guilleminault C, Wei JM, et al. Oropharyngeal and tongue exercises (myofunctional therapy) for snoring: a systematic review and metaanalysis. Eur Arch Otorhinolaryngol. 2018;275(4):849855. doi: 10.1007/s00405-017-4848-5.

4. Schwab RJ, Leinwand SE, Keenan B, Thaler E. Hypoglossal Nerve Stimulation For Obstructive Sleep Apnea: Outcomes And Usage At The University Of Pennsylvania. In. New Technology in Sleep: Diagnostics and Therapeutics 2017 May (pp. A6973-A6973). American Thoracic Society.

5. Bloch KE, Latshang TD, Ulrich S. Patients with Obstructive Sleep Apnea at Altitude. High Alt Med Biol. 2015;16(2):110-116. doi: 10.1089/ ham.2015.0016.

6. Smith PR, Sheikh KL, Costan-Toth C, et al. Eszopiclone and Zolpidem Do Not Affect the Prevalence of the Low Arousal Threshold 
Phenotype. J Clin Sleep Med. 2017;13(1):115-119. Published 2017 Jan 15. doi: 10.5664/jcsm.6402

7. Lee EJ, Cho JH. Meta-Analysis of Obstruction Site Observed With Drug-Induced Sleep Endoscopy in Patients With Obstructive Sleep Apnea. Laryngoscope. 2019;129(5):1235-1243. doi: 10.1002/lary.27320.

8. Fujita S. Obstructive sleep apnea syndrome: pathophysiology, upper airway evaluation and surgical treatment. Ear Nose Throat J. 1993;72(1):6776.

9. Williams R, Patel V, Chen YF, et al. The Upper Airway Nasal Complex: Structural Contribution to Persistent Nasal Obstruction. Otolaryngol Head Neck Surg. 2019;161(1):171-177. doi: 10.1177/0194599819838262.

10. Liu SY, Huon LK, Iwasaki T, et al. Efficacy of Maxillomandibular Advancement Examined with Drug-Induced Sleep Endoscopy and Computational Fluid Dynamics Airflow Modeling. Otolaryngol Head Neck Surg. 2016;154(1):189-195. doi: 10.1177/0194599815611603.

11. Kaprana A, Proimos E, Chimona T, Kiagiadaki D, Papadakis CE. The link between obesity and obstructive sleep apnea syndrome. Arch Hellen Med, 2010; 27(3):539-544.

12. Friedman M, Ibrahim H, Bass L. Clinical staging for sleep-disordered breathing. Otolaryngol Head Neck Surg. 2002;127(1):13-21. doi: 10.1067/ mhn.2002.126477.

13. Conway W, Fujita S, Zorick F, Sicklesteel J, Roehrs T, Wittig R, Roth T. Uvulopalatopharyngoplasty: Oneyear followup. Chest. 1985;88(3):385-7.

14. Li HY. Palatal Surgery for Obstructive Sleep Apnea: From Ablation to Reconstruction. Sleep Med Clin. 2019;14(1):51-58. doi: 10.1016/j.jsmc.2018.10.006.

15. Guilleminault C, Simmons FB, Motta J, et al. Obstructive sleep apnea syndrome and tracheostomy. Long-term follow-up experience. Arch Intern Med. 1981;141(8):985-988.

16. Puccia R, Woodson BT. Palatopharyngoplasty and Palatal Anatomy and Phenotypes for Treatment of Sleep Apnea in the Twenty-first Century. Otolaryngol Clin North Am. 2020;53(3):421-429. doi: 10.1016/j. otc.2020.02.005.

17. Green KK, Woodson BT. Upper Airway Stimulation Therapy. Otolaryngol Clin North Am. 2016;49(6):1425-1431. doi: 10.1016/j. otc.2016.07.010.

18. Haapaniemi JJ, Laurikainen EA, Halme P, Antila J. Long-term results of tracheostomy for severe obstructive sleep apnea syndrome. ORL. 2001;63(3):131-6.

19. Campanini A, De Vito A, Frassineti S, Vicini C. Role of skin-lined tracheotomy in obstructive sleep apnoea syndrome: personal experience. Acta Otorhinolaryngol Ital. 2004;24(2):68-74.

20. Wang M, Liu SY, Zhou B, Li Y, Cui S,
Huang Q. Effect of nasal and sinus surgery in patients with and without obstructive sleep apnea. Acta Otolaryngol. 2019;139(5):467-472. doi: 10.1080/00016489.2019.1575523.

21. Wellman A, Genta PR, Owens RL, et al. Test of the Starling resistor model in the human upper airway during sleep. J Appl Physiol (1985). 2014;117(12):1478-1485. doi: 10.1152/ japplphysiol.00259.2014.

22. Prescinotto R, Haddad FL, Fukuchi I, et al. Impact of upper airway abnormalities on the success and adherence to mandibular advancement device treatment in patients with Obstructive Sleep Apnea Syndrome. Braz J Otorhinolaryngol. 2015;81(6):663670. doi: 10.1016/j.bjorl.2015.08.005.

23. Seren E. A new surgical method of dynamic nasal valve collapse. Arch Otolaryngol Head Neck Surg. 2009;135(10):1010-1014. doi: 10.1001/ archoto.2009.135.

24. Cahali MB. Lateral pharyngoplasty: a new treatment for obstructive sleep apnea hypopnea syndrome. Laryngoscope. 2003;113(11):1961-1968. doi: 10.1097/00005537-200311000-00020.

25. Katsantonis GP, Friedman WH, Krebs FJ 3rd, Walsh JK. Nasopharyngeal complications following uvulopalatopharyngoplasty. Laryngoscope. 1987;97(3 Pt 1):309-314.

26. Oliveira LF, Soter AC, Silva FL, Panerari AU, Rocha-Junior FP, Cedin AC. Partial Resection of the Palate in the treatment of snoring by Quesada \& Perello technique: results. Revista Brasileira de Otorrinolaringologia. 2004;70(1):52-6.

27. Fujita S. UPPP for sleep apnea and snoring. Ear Nose Throat J. 1984;63(5):227-235.

28. Crestinu JM. Intrapalatine resection (IPR) in the treatment of sleep apnea and snoring. Plast Reconstr Surg. 1991;87(3):467-469. doi: 10.1097/00006534199103000-00011.

29. Kamami YV. Outpatient treatment of sleep apnea syndrome with CO2 laser: laser-assisted UPPP. J Otolaryngol. 1994;23(6):395-398.

30. Powell N, Riley R, Guilleminault C, Troell R. A reversible uvulopalatal flap for snoring and sleep apnea syndrome. Sleep. 1996;19(7):593-9.

31. Woodson BT. Transpalatal advancement pharyngoplasty. In Rhinologic and Sleep Apnea Surgical Techniques 2007 (pp. 339-346). Springer, Berlin, Heidelberg.

32. Friedman M, Hwang MS. Z-palatopharyngoplasty. Operative Techniques in Otolaryngology-Head and Neck Surgery. 2015;26(2):90-4.

33. Pang KP, Terris D. Cautery-assisted palatal stiffening operation. Sleep Apnea and Snoring: Surgical and Non-Surgical Therapy. 2008:159.

34. Pang KP, Tan R, Puraviappan P, Terris DJ. Anterior palatoplasty for the treatment of OSA: three-year results. Otolaryngol Head Neck Surg. 2009;141(2):253- 
256. doi: 10.1016/j.otohns.2009.04.020.

35. Mantovani M, Rinaldi V, Torretta S, Carioli D, Salamanca F, Pignataro L. Barbed Roman blinds technique for the treatment of obstructive sleep apnea: how we do it? Eur Arch Otorhinolaryngol. 2016;273(2):517-523. doi: 10.1007/s00405-015-37262.

36. Pang KP, Woodson BT. Expansion sphincter pharyngoplasty: a new technique for the treatment of obstructive sleep apnea. Otolaryngol Head Neck Surg. 2007;137(1):110-114. doi: 10.1016/j. otohns.2007.03.014.

37. Li HY, Lee LA. Relocation pharyngoplasty. Operative Techniques in Otolaryngology-Head and Neck Surgery. 2012;23(1):25-9.

38. Vicini C, Hendawy E, Campanini A, et al. Barbed reposition pharyngoplasty (BRP) for OSAHS: a feasibility, safety, efficacy and teachability pilot study. "We are on the giant's shoulders". Eur Arch Otorhinolaryngol. 2015;272(10):3065-3070. doi: 10.1007/s00405-015-3628-3.

39. Mantovani M, Carioli D, Torretta S, Rinaldi V, Ibba $\mathrm{T}$, Pignataro L. Barbed snore surgery for concentric collapse at the velum: The Alianza technique. J Craniomaxillofac Surg. 2017;45(11):1794-1800. doi: 10.1016/j.jcms.2017.08.007.

40. Barbieri M, Missale F, Incandela F, et al. Barbed suspension pharyngoplasty for treatment of lateral pharyngeal wall and palatal collapse in patients affected by OSAHS. Eur Arch Otorhinolaryngol. 2019;276(6):1829-1835. doi: 10.1007/s00405-01905426-4.

41. Sorrenti G, Pelligra I, Albertini R, Caccamo G, Piccin O. Functional expansion pharyngoplasty: Technical update by unidirectional barbed sutures. Clin Otolaryngol. 2018;43(5):1419-1421. doi: 10.1111/ coa. 13105

42. Woodson BT, Laohasiriwong S. Lingual tonsillectomy and midline posterior glossectomy for obstructive sleep apnea. Operative Techniques in OtolaryngologyHead and Neck Surgery. 2012;23(2):155-61.

43. Gunawardena I, Robinson S, MacKay S, et al. Submucosal lingualplasty for adult obstructive sleep apnea. Otolaryngol Head Neck Surg. 2013;148(1):157-165. doi: 10.1177/0194599812461750.

44. Maturo SC, Mair EA. Submucosal minimally invasive lingual excision (SMILE): Technique for tongue base reduction. Operative Techniques in Otolaryngology-Head and Neck Surgery. 2007;18(1):29-32.

45. MacKay SG, Jefferson N, Grundy L, Lewis R. Coblation-assisted Lewis and MacKay operation (CobLAMO): new technique for tongue reduction in sleep apnoea surgery. J Laryngol Otol. 2013;127(12):1222-1225. doi: 10.1017/ S0022215113002971.
46. Barakate M, Havas T. Lingual tonsillectomy: a review of 5 years experience and evolution of surgical technique. Otolaryngol Head Neck Surg. 2008;139(2):222-227. doi: 10.1016/j. otohns.2008.01.009.

47. D’Agostino MA. Transoral Robotic Partial Glossectomy and Supraglottoplasty for Obstructive Sleep Apnea. Otolaryngol Clin North Am. 2016;49(6):1415-1423. doi: 10.1016/j.otc.2016.07.009.

48. Fujita S, Woodson BT, Clark JL, Wittig R. Laser midline glossectomy as a treatment for obstructive sleep apnea. Laryngoscope. 1991;101(8):805-809. doi: 10.1288/00005537-199108000-00001.

49. Woodson BT, Fujita S. Clinical experience with lingualplasty as part of the treatment of severe obstructive sleep apnea. Otolaryngol Head Neck Surg. 1992;107(1):40-48. doi: 10.1177/019459989210700107.

50. Robinson S. Minimally invasive submucosal glossectomy. In: Sleep apnea and snoring: surgical and non-surgical therapy 2009 (pp. 248-257). Saunders/Elsevier, Philadelphia, PA.

51. Meyer B, Chabolle F, Chouard CH. Surgical treatment of chronic snoring. Description and indications. In; Annales D'oto-laryngologie et de Chirurgie Cervico Faciale: Bulletin de la Societe D'oto-laryngologie des Hopitaux de Paris 1988;105(4):277-282.

52. Li HY, Lee LA, Kezirian EJ. Coblation endoscopic lingual lightening (CELL) for obstructive sleep apnea. Eur Arch Otorhinolaryngol. 2016;273(1):231-236. doi: 10.1007/s00405-014-3475-7.

53. Fernández-Fernández MM, Montes-Jovellar L, Parente Arias PL, Ortega Del Álamo P. TransOral endoscopic UltraSonic Surgery (TOUSS): a preliminary report of a novel robotless alternative to TORS. Eur Arch Otorhinolaryngol. 2015;272(12):3785-3791. doi: 10.1007/s00405-0143423-6.

54. Bahgat A, Bahgat Y, Alzahrani R, Montevecchi F, Cammaroto G, Vicini C. Transoral Endoscopic Coblation Tongue Base Surgery in Obstructive Sleep Apnea: Resection versus Ablation. ORL J Otorhinolaryngol Relat Spec. 2020;82(4):201-208. doi: 10.1159/000506994.

55. Welt S, Maurer JT, Hörmann K, Stuck BA. Radiofrequency surgery of the tongue base in the treatment of snoring-a pilot study. Sleep Breath. 2007;11(1):39-43. doi: 10.1007/s11325-006-0080-z.

56. Coleman J, Bick PA. Suspension sutures for the treatment of obstructive sleep apnea and snoring. Otolaryngol Clin North Am. 1999;32(2):277-285. doi: 10.1016/s0030-6665(05)70130-4.

57. Marchesan IQ, Martinelli RL, Gusmão RJ. Lingual frenulum: changes after frenectomy. Jornal da Sociedade Brasileira de Fonoaudiologia. 2012;24(4):409-12. 
58. Lan MC, Hsu YB, Lan MY, et al. The predictive value of drug-induced sleep endoscopy for CPAP titration in OSA patients. Sleep Breath. 2018;22(4):949-954. doi: 10.1007/s11325-017-1600-8.

59. Gessler EM, Simko EJ, Greinwald JH Jr. Adult laryngomalacia: an uncommon clinical entity. Am J Otolaryngol. 2002;23(6):386-389. doi: 10.1053/ ajot.2002.126322.

60. Gerbino G, Bianchi FA, Verzé L, Ramieri G. Soft tissue changes after maxillo-mandibular advancement in OSAS patients: a three-dimensional study. J Craniomaxillofac Surg. 2014;42(1):66-72. doi: 10.1016/j.jcms.2013.02.004

61. Sittitavornwong S, Waite PD, Shih AM, Koomullil R, Ito Y, Cheng GC, Wang D. Evaluation of obstructive sleep apnea syndrome by computational fluid dynamics. In: Seminars in Orthodontics, WB Saunders. 2009;15(2):105-131.

62. Askar SM, El-Anwar MW, Awad A. Expansion Hyoidthyroidpexy: Combined Hyoid Surgery Techniques for Obstructive Sleep Apnea: All in One. Otolaryngol Head Neck Surg. 2019;160(2):355-358. doi: 10.1177/0194599818815658.

63. Tantawy AA, Askar SM, Amer HS, Awad A, El-Anwar MW. Hyoid Bone Suspension as a Part of Multilevel Surgery for Obstructive Sleep Apnea Syndrome. Int Arch Otorhinolaryngol. 2018;22(3):266270. doi: 10.1055/s-0037-1607227.

64. Krespi YP, Kacker A. Hyoid suspension for obstructive sleep apnea. Operative Techniques in Otolaryngology-Head and Neck Surgery. 2002;13(2):144-9.

65. Benoist LB, van Maanen JP, de Vries N. Hyoid suspension: hyothyroid and hyomandibular options. Operative Techniques in Otolaryngology-Head and
Neck Surgery. 2015;26(4):178-82.

66. Vinha PP, Thuler ER, de Mello-Filho FV. Effects of surgically assisted rapid maxillary expansion on the modification of the pharynx and hard palate and on obstructive sleep apnea, and their correlations. J Craniomaxillofac Surg. 2020;48(4):339-348. doi: 10.1016/j.jcms.2020.02.007.

67. Yoon A, Guilleminault C, Zaghi S, Liu SY. Distraction Osteogenesis Maxillary Expansion (DOME) for adult obstructive sleep apnea patients with narrow maxilla and nasal floor. Sleep Med. 2020;65:172-176. doi: 10.1016/j.sleep.2019.06.002.

68. Li K, Quo S, Guilleminault C. Endoscopically-assisted surgical expansion (EASE) for the treatment of obstructive sleep apnea. Sleep Med. 2019;60:53-59. doi: 10.1016/j.sleep.2018.09.008.

69. Hayes JL. Rapid maxillary expansion. Am J Orthod Dentofacial Orthop. 2006;130(4):432-434. doi: 10.1016/j.ajodo.2006.08.006.

70. Carlson C, Sung J, McComb RW, Machado AW, Moon W. Microimplant-assisted rapid palatal expansion appliance to orthopedically correct transverse maxillary deficiency in an adult. Am J Orthod Dentofacial Orthop. 2016;149(5):716-728. doi: 10.1016/j.ajodo.2015.04.043.

71. Sarkhosh K, Switzer NJ, El-Hadi M, Birch DW, Shi X, Karmali S. The impact of bariatric surgery on obstructive sleep apnea: a systematic review. Obes Surg. 2013;23(3):414-423. doi: 10.1007/s11695-0120862-2.

72. Woodson BT, Strohl KP, Soose RJ, et al. Upper Airway Stimulation for Obstructive Sleep Apnea: 5-Year Outcomes. Otolaryngol Head Neck Surg. 2018;159(1):194-202. doi: $10.1177 / 0194599818762383$. 